\title{
Existence and uniqueness of solutions to fractional differential equations in the frame of generalized Caputo fractional derivatives
}

\author{
Y.Y. Gambo ', R. Ameen ${ }^{2}$, F. Jarad ${ }^{3 *}$ and T. Abdeljawad ${ }^{4}$
}

${ }^{\text {*Correspondence: }}$

fahd@cankaya.edu.tr

${ }^{3}$ Department of Mathematics,

Faculty of Arts and Sciences,

Cankaya University, Ankara, Turkey

Full list of author information is

available at the end of the article

\section{Springer}

\begin{abstract}
The generalized Caputo fractional derivative is a name attributed to the Caputo version of the generalized fractional derivative introduced in Jarad et al. (J. Nonlinear Sci. Appl. 10:2607-2619, 2017). Depending on the value of $\rho$ in the limiting case, the generality of the derivative is that it gives birth to two different fractional derivatives. However, the existence and uniqueness of solutions to fractional differential equations with generalized Caputo fractional derivatives have not been proven. In this paper, Cauchy problems for differential equations with the above derivative in the space of continuously differentiable functions are studied. Nonlinear Volterra type integral equations of the second kind corresponding to the Cauchy problem are presented. Using Banach fixed point theorem, the existence and uniqueness of solution to the considered Cauchy problem is proven based on the results obtained.
\end{abstract}

Keywords: Generalized Caputo fractional derivative; Existence and uniqueness; Cauchy problem

\section{Introduction}

The fractional calculus is the branch of mathematics that studies the integration and differentiation of real or complex orders. Even though this calculus is old, it has been gaining astounding popularity for the recent decades only. This is due to its numerous seemingly diverse applications [2-8]. The most interesting speciality of the fractional operators is that there are many of these operators. This enables a researcher to choose the most suitable operator in order to describe the dynamics in a real world problem.

The fractional calculus was bounded up with fractional integrals obtained by iterating an integral to get the $n$th order integral and then replacing $n$ by any number, and then by using the classical method the corresponding derivatives were defined (see, for example, $[1,9-14])$. However, for the sake of better description of real world phenomena, some scientists discovered new fractional operators with nonlocal and nonsingular kernels using the limiting process with the help of the Dirac delta function. For such operators, we refer to [15-17]. Other types of new fractional derivatives can be found in [18-21].

On the other hand, the existence and uniqueness of solutions are among the most important qualitative properties of differential equations. The existence and uniqueness of solutions of differential equations involving the fractional derivatives were tackled by many researchers (see [22-26] and the references therein).

(c) The Author(s) 2018. This article is distributed under the terms of the Creative Commons Attribution 4.0 International License (http://creativecommons.org/licenses/by/4.0/), which permits unrestricted use, distribution, and reproduction in any medium, provided you give appropriate credit to the original author(s) and the source, provide a link to the Creative Commons license, and indicate if changes were made. 
This paper studies fractional Cauchy problems with left generalized Caputo fractional derivatives in the space of continuously differentiable functions and proves the existence and uniqueness of solutions to these problems. We consider the following Cauchy problem:

$$
\left({ }_{a}^{C} D^{\alpha, \rho} x\right)(t)=h\left[t, x(t),\left({ }_{a}^{C} D^{\alpha_{1}, \rho} x\right)(t), \ldots,\left({ }_{a}^{C} D^{\alpha_{m}, \rho} x\right)(t)\right]
$$

subject to the initial conditions

$$
\left(\gamma^{k} x\right)(a)=d_{k}, \quad d_{k} \in \mathbb{R}(k=0,1, \ldots, n-1),
$$

where $\rho \in \mathbb{R}^{+}, n=[\alpha]+1, \alpha_{j} \in(j-1, j], j=1,2, \ldots, m<n ; \alpha_{0}=0, \gamma=t^{1-\rho} \frac{d}{d t}$ and ${ }_{a}^{C} D^{\alpha_{j}, \rho}$ denotes the generalized Caputo fractional differential operator of order $\alpha_{j}$.

As part of the main work, nonlinear Volterra type integral equations of the second kind corresponding to the Cauchy problems are shown and, subsequently, Banach fixed point theorem is applied. But before we start, let us recall some definitions from the fractional calculus [2-4].

The left Riemann-Liouville fractional integral of order $\alpha, \Re(\alpha)>0$ is defined by

$$
\left({ }_{a} I^{\alpha} f\right)(t)=\frac{1}{\Gamma(\alpha)} \int_{a}^{t}(t-u)^{\alpha-1} f(u) d u .
$$

The right Riemann-Liouville fractional integral of order $\alpha, \Re(\alpha)>0$ is defined by

$$
\left(I_{b}^{\alpha} f\right)(t)=\frac{1}{\Gamma(\alpha)} \int_{t}^{b}(u-t)^{\alpha-1} f(u) d u
$$

The left Riemann-Liouville fractional derivative of order $\alpha, \Re(\alpha) \geq 0$ is given as

$$
\left({ }_{a} D^{\alpha} f\right)(t)=\frac{d^{n}}{d t^{n}}\left({ }_{a} I^{n-\alpha} f\right)(t)=\frac{\frac{d^{n}}{d t^{n}}}{\Gamma(n-\alpha)} \int_{a}^{t}(t-u)^{n-\alpha-1} f(u) d u, \quad n=[\Re(\alpha)]+1 .
$$

The right Riemann-Liouville fractional derivative of order $\alpha, \Re(\alpha) \geq 0$ reads as follows:

$$
\begin{aligned}
\left(D_{b}^{\alpha} f\right)(t) & =(-1)^{n} \frac{d^{n}}{d t^{n}}\left(I_{b}^{n-\alpha} f\right)(t) \\
& =\frac{(-1)^{n} \frac{d^{n}}{d t^{n}}}{\Gamma(n-\alpha)} \int_{t}^{b}(u-t)^{n-\alpha-1} f(u) d u, \quad n=[\Re(\alpha)]+1 .
\end{aligned}
$$

The left Caputo fractional derivative of order $\alpha, \Re(\alpha) \geq 0$ has the form

$$
\left({ }_{a}^{C} D^{\alpha} f\right)(t)=\left({ }_{a} I^{n-\alpha} f^{(n)}\right)(t)=\frac{1}{\Gamma(n-\alpha)} \int_{a}^{t}(t-u)^{\alpha-1} f^{(n)}(u) d u, \quad n=[\Re(\alpha)]+1 .
$$

The right Caputo fractional derivative of order $\alpha, \Re(\alpha) \geq 0$ reads as follows:

$$
\begin{aligned}
\left({ }^{C} D_{b}^{\alpha} f\right)(t) & =\left(I_{b}^{n-\alpha}(-1)^{n} f^{(n)}\right)(t) \\
& =\frac{(-1)^{n}}{\Gamma(n-\alpha)} \int_{t}^{b}(u-t)^{n-\alpha-1} f^{(n)}(u) d u, \quad n=[\Re(\alpha)]+1 .
\end{aligned}
$$


The Hadamard type fractional integrals and derivatives were introduced in [9].

The left Hadamard fractional integral of order $\alpha, \Re(\alpha)>0$ has the following form:

$$
\left({ }_{a} \mathcal{J}^{\alpha} f\right)(t)=\frac{1}{\Gamma(\alpha)} \int_{a}^{t}(\log t-\log u)^{\alpha-1} f(u) \frac{d u}{u} .
$$

The right Hadamard fractional integral of order $\alpha, \Re(\alpha)>0$ is defined by

$$
\left(\mathcal{J}_{b}^{\alpha} f\right)(t)=\frac{1}{\Gamma(\alpha)} \int_{t}^{b}(\log u-\log t)^{\alpha-1} f(u) \frac{d u}{u} .
$$

The left Hadamard fractional derivative of order $\alpha, \Re(\alpha) \geq 0$ is given by

$$
\begin{aligned}
\left({ }_{a} \mathcal{D}^{\alpha} f\right)(t) & =\left(t \frac{d}{d t}\right)^{n}\left(\mathcal{J}^{n-\alpha} f\right)(t) \\
& =\frac{\left(t \frac{d}{d t}\right)^{n}}{\Gamma(\alpha)} \int_{a}^{t}(\log t-\log u)^{\alpha-1} f(u) \frac{d u}{u}, \quad n=[\Re(\alpha)]+1 .
\end{aligned}
$$

The right Hadamard fractional derivative of order $\alpha, \Re(\alpha) \geq 0$ reads as follows:

$$
\begin{aligned}
\left(\mathcal{D}_{b}^{\alpha} f\right)(t) & =\left(-t \frac{d}{d t}\right)^{n}\left(\mathcal{J}_{b}^{n-\alpha} f\right)(t) \\
& =\frac{\left(-t \frac{d}{d t}\right)^{n}}{\Gamma(\alpha)} \int_{t}^{b}(\log u-\log t)^{\alpha-1} f(u) \frac{d u}{u}, \quad n=[\Re(\alpha)]+1 .
\end{aligned}
$$

The left Caputo-Hadamard fractional derivative of order $\alpha, \Re(\alpha) \geq 0$ is presented as $[12,13]$

$$
\left({ }_{a}^{C} \mathcal{D}^{\alpha} f\right)(t)={ }_{a} \mathcal{D}^{\alpha}\left[f(u)-\sum_{k=0}^{n-1} \frac{\delta^{k} f(a)}{k !}\left(\log \frac{u}{a}\right)^{k}\right](t), \quad \delta=t \frac{d}{d t},
$$

and in the space $A C_{\delta}^{n}[a, b]=\left\{g:[a, b] \rightarrow \mathbb{C}: \delta^{n-1}[g(t)] \in A C[a, b]\right\}$ equivalently by

$$
\left({ }_{a}^{C} \mathcal{D}^{\alpha} f\right)(t)=\left({ }_{a} \mathcal{J}^{n-\alpha}\left(t \frac{d}{d t}\right)^{n} f\right)(t), \quad n=[\Re(\alpha)]+1 .
$$

The right Caputo-Hadamard fractional derivative of order $\alpha, \Re(\alpha) \geq 0>0$ is defined by $[12,13]$

$$
\left({ }_{a}^{C} \mathcal{D}^{\alpha} f\right)(t)={ }_{a} \mathcal{D}^{\alpha}\left[f(u)-\sum_{k=0}^{n-1} \frac{(-1)^{k} \delta^{k} f(b)}{k !}\left(\log \frac{b}{u}\right)^{k}\right](t),
$$

and in the space $A C_{\delta}^{n}[a, b]$ equivalently by

$$
\left({ }^{C} \mathcal{D}_{b}^{\alpha} f\right)(t)=\left(\mathcal{J}_{b}^{n-\alpha}\left(-t \frac{d}{d t}\right)^{n} f\right)(t)
$$

For $a<b, c \in \mathbb{R}$, and $1 \leq p<\infty$, define the measurable Lebesgue function space

$$
X_{c}^{p}(a, b)=\left\{f:[a, b] \rightarrow \mathbb{R}:\|f\|_{X_{c}^{p}}=\left(\int_{a}^{b}\left|t^{c} f(t)\right|^{p} \frac{d t}{t}\right)^{1 / p}<\infty\right\} .
$$


For $p=\infty,\|f\|_{X_{c}^{p}}=\operatorname{ess} \sup _{a \leq t \leq b}\left[t^{c}|f(t)|\right]$. The generalized left and right fractional integrals of order $\alpha, \Re(\alpha)>0$ are defined in [10] as

$$
\left({ }_{a} I^{\alpha, \rho} f\right)(t)=\frac{1}{\Gamma(\alpha)} \int_{a}^{t}\left(\frac{t^{\rho}-u^{\rho}}{\rho}\right)^{\alpha-1} f(u) \frac{d u}{u^{1-\rho}}
$$

and

$$
\left(I_{b}^{\alpha, \rho} f\right)(t)=\frac{1}{\Gamma(\alpha)} \int_{t}^{b}\left(\frac{u^{\rho}-t^{\rho}}{\rho}\right)^{\alpha-1} f(u) \frac{d u}{u^{1-\rho}}
$$

respectively.

One can notice that when $\rho=1$, the integrals in (17) and (18) reduce to the integrals in (2) and (3), respectively. Moreover, when one takes the limits of the integrals in (17) and (18) as $\rho \rightarrow 0$, the Hadamard fractional integrals in (9) and (10) are obtained, respectively.

The left and right generalized fractional derivatives of order $\alpha, \Re(\alpha) \geq 0$ are defined by (see [11])

$$
\left({ }_{a} D^{\alpha, \rho} f\right)(t)=\gamma^{n}\left({ }_{a} I^{n-\alpha, \rho} f\right)(t)=\frac{\gamma^{n}}{\Gamma(n-\alpha)} \int_{a}^{t}\left(\frac{t^{\rho}-u^{\rho}}{\rho}\right)^{n-\alpha-1} f(u) \frac{d u}{u^{1-\rho}}
$$

and

$$
\left(D_{b}^{\alpha, \rho} f\right)(x)=(-\gamma)^{n}\left({ }_{a} I^{n-\alpha, \rho} f\right)(t)=\frac{(-\gamma)^{n}}{\Gamma(n-\alpha)} \int_{t}^{b}\left(\frac{u^{\rho}-t^{\rho}}{\rho}\right)^{n-\alpha-1} f(u) \frac{d u}{u^{1-\rho}}
$$

respectively, where $\gamma=t^{1-\rho} \frac{d}{d t}$. Putting $\rho=1$ in (19) and (20), one gets the RiemannLiouville fractional derivatives (5) and (6); and letting $\rho$ tend to 0 , one gets the Hadamard fractional derivatives (11) and (12).

For the functions in $A C_{\gamma}^{n}[a, b]=\left\{f:[a, b] \rightarrow \mathbb{C}\right.$ and $\left.\gamma^{n-1} f \in A C[a, b], \gamma=t^{1-\rho} \frac{d}{d t}\right\}$ and $C_{\gamma}^{n}[a, b]=\left\{f:[a, b] \rightarrow \mathbb{C}\right.$ and $\left.\gamma^{n-1} f \in C[a, b], \gamma=t^{1-\rho} \frac{d}{d t}\right\}$, the left and right generalized Caputo fractional derivatives of order $\alpha, \Re(\alpha)>0$ are given respectively as in [1] as follows:

$$
{ }_{a}^{C} D^{\alpha, \rho} f(t)=\frac{1}{\Gamma(n-\alpha)} \int_{a}^{t}\left(\frac{t^{\rho}-u^{\rho}}{\rho}\right)^{n-\alpha-1} \frac{\left(\gamma^{n} f\right)(u) d u}{u^{1-\rho}}={ }_{a} I^{n-\alpha, \rho}\left(\gamma^{n} f\right)(t)
$$

and

$$
\begin{aligned}
{ }^{C} D_{b}^{\alpha, \rho} f(t) & =\frac{1}{\Gamma(n-\alpha)} \int_{t}^{b}\left(\frac{u^{\rho}-t^{\rho}}{\rho}\right)^{n-\alpha-1} \frac{(-1)^{n}\left(\gamma^{n} f\right)(u) d u}{u^{1-\rho}} \\
& =I_{b}^{n-\alpha, \rho}\left((-1)^{n} \gamma^{n} f\right)(t) .
\end{aligned}
$$

It can be observed that (21) becomes the left Caputo derivative (7) when one replaces $\rho$ by 1 and the left Caputo-Hadamard derivative (14) if one takes the limit as $\rho$ approaches 0. The same relation holds for (22) and (8), and (22) and (16).

This paper is organized as follows. In Sect. 2 we present definitions, notations, and lemmas that will be used in this work. In Sect. 3 we present the Cauchy type problem for which the existence and uniqueness are considered. 


\section{Auxiliary results}

Let $C^{n}([a, b], \mathbb{C})$ be the Banach space of all continuously differentiable functions from $[a, b]$ to $\mathbb{C}$. We recall the space $C_{\gamma}^{n}[a, b]$ and define the weighted spaces $C_{\epsilon, \rho}[a, b], C_{\gamma, \epsilon}^{n}[a, b]$, and $C_{\gamma}^{\alpha, r}[a, b]$ of a function $f$. For $n-1<\Re(\alpha) \leq n, 0 \leq \Re(\epsilon)<1$, and $\rho \in \mathbb{R}^{+}$, we define

$$
\begin{aligned}
& C_{\gamma}^{n}[a, b]=\left\{f:[a, b] \rightarrow \mathbb{C} \text { s.t. } \gamma^{n} f \in C[a, b]\right\} . \\
& C_{\epsilon, \rho}[a, b]=\left\{f:\left(\frac{t^{\rho}-a^{\rho}}{\rho}\right)^{\epsilon} f(x) \in C[a, b]\right\} ; \quad C_{0, \rho}[a, b]=C[a, b] \text { for } \rho \neq 0
\end{aligned}
$$

endowed with the norm

$$
\begin{aligned}
& \|f\|_{C_{\epsilon, \rho}}=\left\|\left(\frac{t^{\rho}-a^{\rho}}{\rho}\right)^{\epsilon} f(t)\right\|_{C}=\max _{t \in[a, b]}\left|\left(\frac{t^{\rho}-a^{\rho}}{\rho}\right)^{\epsilon} f(t)\right| . \\
& C_{\epsilon, \rho}[a, b]=\left\{f:\left(\log \frac{x}{a}\right)^{\epsilon} f(x) \in C[a, b]\right\} ; \quad C_{0, \rho}[a, b]=C[a, b] \quad \text { for } \rho=0
\end{aligned}
$$

endowed with the norm

$$
\|f\|_{C_{\epsilon, \rho}}=\|f\|_{C_{\epsilon, \log }}=\left\|\left(\log \frac{x}{a}\right)^{\epsilon} f(x)\right\|_{C}=\max _{x \in[a, b]}\left|\left(\log \frac{x}{a}\right)^{\epsilon} f(x)\right| .
$$

The space $C_{\gamma, \epsilon}^{n}[a, b]$ is defined by

$$
C_{\gamma, \epsilon}^{n}[a, b]=\left\{f: \gamma^{n-1} f \in C[a, b] \text { and } \gamma^{n} f \in C_{\epsilon, \rho}[a, b], \rho>0\right\}
$$

endowed with the norm

$$
\begin{aligned}
& \|f\|_{C_{\gamma, \epsilon}^{n}}=\sum_{k=0}^{n-1}\left\|\gamma^{k} f\right\|_{C}+\left\|\gamma^{n} f\right\|_{C_{\epsilon, \rho}}, \\
& (\epsilon=0 \Rightarrow)\|f\|_{C_{\gamma}^{n}}=\sum_{k=0}^{n} \max _{x \in[a, b]}\left|\gamma^{k} f(x)\right|, \quad \rho>0 .
\end{aligned}
$$

For $0 \leq \epsilon<1, \epsilon \leq \alpha$, we define $C_{\gamma}^{\alpha, r}[a, b]$ by

$$
\begin{aligned}
& C_{\gamma, \epsilon}^{\alpha, r}[a, b]=\left\{f \in C_{\gamma}^{r}[a, b]:\left({ }_{a}^{C} D^{\alpha, \rho} f\right) \in C_{\epsilon, \rho}[a, b], r \in \mathbb{N}\right\}, \\
& C_{\gamma}^{r, r}[a, b]=C_{\gamma, \epsilon}^{r}[a, b],
\end{aligned}
$$

where we use $C_{\gamma, \epsilon}^{\alpha, 0}[a, b]=C_{\gamma, \epsilon}^{\alpha}[a, b]$ and $C_{0, \rho}[a, b]=C_{\gamma}^{0}[a, b]=C[a, b]$. The generalized fractional integrals and generalized fractional derivatives satisfy the following semigroup properties.

Lemma 2.1 ([10]) For a function $f$ defined on $[a, b]$, we have

$$
\left({ }_{a} I^{\alpha, \rho}\left({ }_{a} I^{\beta, \rho} f\right)\right)(t)=\left({ }_{a} I^{\alpha+\beta, \rho} f\right)(t), \quad \Re(\alpha)>0, \Re(\beta)>0 .
$$


Lemma 2.2 ([1]) For a function $f \in X_{c}^{p}(a, b), \rho \geq c$, we have

$$
\left({ }_{a} D^{\alpha, \rho}\left({ }_{a} I^{\beta, \rho} f\right)\right)(t)=\left({ }_{a} I^{\beta-\alpha, \rho} f\right)(t), \quad \beta>\alpha>0,
$$

holds almost everywhere on $[a, b]$. When $\alpha=\beta$, we have $\left({ }_{a} D^{\alpha, \rho}\left({ }_{a} I^{\alpha, \rho} f\right)\right)(t)=f(t)$ almost everywhere.

Lemma $2.3([1])$ Let $\Re(\alpha>0), n=[\Re(\alpha)]+1$, and $\Re(\beta)>0$, then

$$
{ }_{a}^{C} D^{\alpha, \rho}\left(\frac{x^{\rho}-a^{\rho}}{\rho}\right)^{\beta-1}=\frac{\Gamma(\beta)}{\Gamma(\beta-\alpha)}\left(\frac{x^{\rho}-a^{\rho}}{\rho}\right)^{\beta-\alpha-1}, \quad \Re(\beta)>n .
$$

For $k=0,1, \ldots, n-1$,

$$
{ }_{a}^{C} D^{\alpha, \rho}\left(\frac{x^{\rho}-a^{\rho}}{\rho}\right)^{k}=0
$$

Lemma 2.4 ([1]) Let $\alpha \in \mathbb{C}, n=[\Re(\alpha)]+1, f \in A C_{\gamma}^{n}[a, b]$, or $C_{\gamma}^{n}[a, b]$. Then

$$
\left({ }_{a} I^{\alpha, \rho}\left({ }_{a}^{C} D^{\alpha, \rho} f\right)\right)(x)=f(x)-\sum_{k=0}^{n-1} \frac{\left(\gamma^{k} f\right)(a)}{k !}\left(\frac{x^{\rho}-a^{\rho}}{\rho}\right)^{k} .
$$

Lemma 2.5 ([1]) Assume $\rho>0$. Then the space $C_{\gamma}^{n}[a, b]$ consists of those and only those functions $f$ which are represented in the form

$$
f(t)=\frac{a}{(n-1) !} \int_{a}^{t}\left(\frac{t^{\rho}-u^{\rho}}{\rho}\right)^{n-1} \frac{\left(\gamma^{n} f\right)(u)}{u^{1-\rho}} d u+\sum_{k=0}^{n-1} \frac{\left(\gamma^{k} f\right)(a)}{k !}\left(\frac{t^{\rho}-a^{\rho}}{\rho}\right)^{k} .
$$

Theorem 2.6 (Banach fixed point theorem) Let $(X, d)$ be a nonempty complete metric space, and let $0 \leq \lambda<1$. If $T: X \rightarrow X$ is a mapping such that for every $x, \tilde{x} \in X$, the relation

$$
d(T x, T \tilde{x}) \leq \lambda d(x, \tilde{x})
$$

holds, then the operator $T$ has a unique defined fixed point $x^{*} \in X$. Moreover, if $T^{k}(k \in \mathbb{N})$ is the sequence defined by

$$
\left\{\begin{array}{l}
T^{k}=T T^{k-1}, \quad k \in \mathbb{N} \backslash\{1\} \\
T^{1}=T
\end{array}\right.
$$

then, for any $x_{0} \in X,\left\{T^{k} x_{0}\right\}_{k=1}^{k=\infty}$ converges to the above fixed point $x^{*}$.

Definition 2.1 Let $m \in \mathbb{N}, G \subset \mathbb{R}^{m},[a, b] \subset \mathbb{R}$ and $f:[a, b] \times G \rightarrow \mathbb{R}$ be a function such that, for any $\left(x_{1}, \ldots, x_{m}\right),\left(\tilde{x}_{1}, \ldots, \tilde{x}_{m}\right) \in G, f$ satisfies the generalized Lipschitzian condition:

$$
\begin{aligned}
& \left|f\left[t, x_{1}, \ldots, x_{m}\right]-f\left[t, \tilde{x}_{1}, \ldots, \tilde{x}_{m}\right]\right| \leq A_{1}\left|x_{1}-\tilde{x}_{1}\right|+\cdots+A_{m}\left|x_{m}-\tilde{x}_{m}\right|, \\
& A_{j} \geq 0, j=1, \ldots, m .
\end{aligned}
$$


In particular, $f$ satisfies the Lipschitzian condition with respect to the second variable if, for all $t \in(a, b]$ and for any $x, \tilde{x} \in G$, one has

$$
|f[t, x]-f[t, \tilde{x}]| \leq A|x-\tilde{x}|, \quad A>0 .
$$

Lemma 2.7 ([27]) Let $0<a<b<\infty, \alpha>0$, and $0 \leq \epsilon<1$, then

(a) If $0<\alpha<\epsilon$, then ${ }_{a} I^{\alpha, \rho}$ is bounded from $C_{\epsilon, \rho}[a, b]$ into $C_{\epsilon-\alpha, \rho}[a, b]$ :

$$
\left\|a^{\alpha, \rho} f\right\|_{C_{\epsilon-\alpha, \rho}} \leq\left(\frac{b^{\rho}-a^{\rho}}{\rho}\right)^{\alpha} \frac{\Gamma(1-\epsilon)}{\Gamma(1+\alpha-\epsilon)}\|f\|_{C_{\epsilon, \rho}}
$$

(b) If $\alpha \geq \epsilon$, then ${ }_{a} I^{\alpha, \rho}$ is bounded from $C_{\epsilon, \rho}[a, b]$ into $C[a, b]$ :

$$
\|\|_{a} I^{\alpha, \rho} f\left\|_{C} \leq\left(\frac{b^{\rho}-a^{\rho}}{\rho}\right)^{\alpha-\epsilon} \frac{\Gamma(1-\epsilon)}{\Gamma(1+\alpha-\epsilon)}\right\| f \|_{C_{\epsilon, \rho}} ;
$$

(c) The fractional integral operator ${ }_{a} I^{\alpha, \rho}$ represents a mapping from $C[a, b]$ to $C[a, b]$ and

$$
\left\|a^{\alpha, \rho} f\right\|_{C} \leq \frac{1}{\Gamma(\alpha+1)}\left(\frac{b^{\rho}-a^{\rho}}{\rho}\right)^{\alpha}\|f\|_{C} .
$$

\section{Cauchy type problem generalized with generalized Caputo fractional derivative}

We now present the existence and uniqueness of solutions to the Cauchy problem (1)-(2) in the space $C_{\gamma}^{\alpha, r}[a, b]$ for a nonlinear fractional differential equation with generalized Caputo fractional derivative. We denote $h\left[t, x(t),\left({ }_{a}^{C} D^{\alpha_{1}, \rho} x\right)(t), \ldots,\left({ }_{a}^{C} D^{\alpha_{m}, \rho} x\right)(t)\right]$ by $h[t, \psi(t, x)]$ for the sake of simplicity.

The Volterra type integral equation corresponding to problem (1)-(2) can be written as

$$
x(t)=\sum_{j=0}^{n-1} \frac{d_{j}}{j !}\left(\frac{t^{\rho}-a^{\rho}}{\rho}\right)^{j}+\frac{1}{\Gamma(\alpha)} \int_{a}^{x}\left(\frac{t^{\rho}-\tau^{\rho}}{\rho}\right)^{\alpha-1} h[\tau, \psi(\tau, x)] \frac{d \tau}{\tau^{1-\rho}} d t, \quad t>a .
$$

Theorem 3.1 Let $\alpha>0, n=[\alpha]+1$, and $\alpha_{j} \in \mathbb{R}(j=0, \ldots, m)$ such that

$$
0=\alpha_{0}<\alpha_{1}<\cdots<\alpha_{m}<n-1 .
$$

Let $G \in \mathbb{R}^{m+1}$ be open subsets, and let $h:(a, b] \times G \rightarrow \mathbb{R}$ be a function such that $h\left[t, x, x_{1}, \ldots, x_{m}\right] \in C_{\epsilon, \rho}[a, b]$ for arbitrary $x, x_{1}, \ldots, x_{m} \in C_{\epsilon, \rho}[a, b]$, and the Lipschitz condition (38) is satisfied.

(a) If $x \in C_{\gamma}^{\alpha, n-1}[a, b]$, then $x$ satisfies relations (1)-(2) if and only if $x$ satisfies equation $(44)$.

(b) If $0<\alpha<1$, then $x \in C_{\gamma}^{\alpha}[a, b]$ satisfies the relations

$$
\left({ }_{a}^{C} D^{\alpha, \rho} x\right)(t)=h[t, \psi(t, x)], \quad x(a)=d_{0}, d_{0} \in \mathbb{R}
$$

if and only if $x$ satisfies the equation

$$
x(t)=d_{0}+\left({ }_{a} I^{\alpha, \rho} h[\tau, \psi(\tau, x)]\right)(t), \quad t>a .
$$


Proof Let $\alpha \in(n-1, n)$ and suppose $x \in C_{\gamma}^{n-1}[a, b]$ satisfies equations (1)-(2). According to Definition 3.1 in [1],

$$
\left({ }_{a}^{C} D^{\alpha, \rho} x\right)(t)={ }_{a} D^{\alpha, \rho}\left[x(\tau)-\sum_{k=0}^{n-1} \frac{\left(\gamma^{k} x\right)(a)}{k !}\left(\frac{t^{\rho}-a^{\rho}}{\rho}\right)^{k}\right](t) .
$$

Then we have that $\left({ }_{a}^{C} D^{\alpha, \rho} x\right)(t) \in C_{\epsilon, \rho}[a, b]$, which implies

$$
\gamma_{a}^{n} I^{n-\alpha, \rho}\left[x(\tau)-\sum_{k=0}^{n-1} \frac{\left(\gamma^{k} x\right)(a)}{k !}\left(\frac{t^{\rho}-a^{\rho}}{\rho}\right)^{k}\right](t) \in C_{\epsilon, \rho}[a, b] .
$$

Then, using (1), (2) and Lemma 2.4, we have

$$
\begin{aligned}
x(t) & =\left({ }_{a} I^{\alpha, \rho}\left({ }_{a}^{C} D^{\alpha, \rho} x\right)\right)(t)+\sum_{k=0}^{n-1} \frac{\left(\gamma^{k} x\right)(a)}{k !}\left(\frac{t^{\rho}-a^{\rho}}{\rho}\right)^{k} \\
& ={ }_{a} I^{\alpha, \rho} h[\tau, \psi(\tau, x)](t)+\sum_{k=0}^{n-1} \frac{d_{k}}{k !}\left(\frac{t^{\rho}-a^{\rho}}{\rho}\right)^{k}, \quad t>a .
\end{aligned}
$$

This means $x \in C_{\gamma}^{n-1}[a, b]$ satisfies (44).

To prove the sufficiency, let $x \in C_{\gamma}^{n-1}[a, b]$ satisfy (44). Then

$$
{ }_{a} I^{\alpha, \rho} h[\tau, \psi(\tau, x)](t)=x(t)-\sum_{j=0}^{n-1} \frac{d_{j}}{j !}\left(\frac{t^{\rho}-a^{\rho}}{\rho}\right)^{j} .
$$

Taking the generalized fractional derivative ${ }_{a} D^{\alpha, \rho}$ of both sides of this relation and considering the conditions for $h$, we obtain

$$
{ }_{a} D^{\alpha, \rho}{ }_{a} I^{\alpha, \rho} h[\tau, \psi(\tau, x)](t)={ }_{a} D^{\alpha, \rho}\left[x(t)-\sum_{j=0}^{n-1} \frac{d_{j}}{j !}\left(\frac{t^{\rho}-a^{\rho}}{\rho}\right)^{j}\right]=\left({ }_{a}^{C} D^{\alpha, \rho} x\right)(t),
$$

where we have used Definition 3.1 in [1]. Thus, $x \in C_{\gamma}^{n-1}[a, b]$ satisfies (1).

On the other hand, applying $\gamma^{k}, k=0,1, \ldots, n-1$, to both sides of (44) gives

$$
\begin{aligned}
\gamma^{k} x(t) & =\sum_{j=k}^{n-1} \frac{d_{j}}{(j-k) !}\left(\frac{t^{\rho}-a^{\rho}}{\rho}\right)^{j-k}+\gamma^{k}{ }_{a} I^{\alpha, \rho} h[\tau, \psi(\tau, x)](t), \quad t>a \\
& =\sum_{j=k}^{n-1} \frac{d_{j}}{(j-k) !}\left(\frac{t^{\rho}-a^{\rho}}{\rho}\right)^{j-k}+{ }_{a} D^{\alpha, \rho} h[\tau, \psi(\tau, x)](t), \quad t>a .
\end{aligned}
$$

Since the fractional derivative at an end point is zero, i.e., $\left({ }_{a} D^{\alpha, \rho} f\right)(a)=0$, then letting $\tau \rightarrow a$ we obtain

$$
\left(\gamma^{k} x\right)(a)=d_{k}, \quad k=0,1, \ldots, n-1 .
$$

Thus, if $x$ satisfies (44), then it also satisfies the initial condition (2). Hence $x \in C_{\gamma}^{n-1}[a, b]$ satisfies the Cauchy problem (1)-(2). 
The second part of the theorem can be proven analogously. Note that since $0<\alpha<1$, this implies $n=1$, and therefore the term $\sum_{k=0}^{n-1} \frac{\left(\gamma^{k} x\right)(a)}{k !}\left(\frac{t^{\rho}-a^{\rho}}{\rho}\right)^{k}$ reduces to $x(a)$.

Theorem 3.2 Let $\alpha \in \mathbb{R}, n=[\alpha]+1,0 \leq \epsilon<1$ such that $\epsilon \leq \alpha$. Let $\alpha_{j}>0, j=1, \ldots, m$, satisfying (25). Suppose $G$ is an open set in $\mathbb{R}^{m+1}$ and $h:(a, b] \times G \rightarrow \mathbb{R}$ is a function such that $h\left[t, x, x_{1}, \ldots, x_{m}\right] \in C_{\epsilon, \rho}[a, b]$ and the Lipschitz condition (39) is satisfied.

(a) If $n-1<\alpha<n$, then there exists a unique solution $x$ to problem (22)-(23) in the space $C_{\gamma, \epsilon}^{\alpha, n-1}[a, b]$.

(b) If $0<\alpha<1$, then there exists a unique solution $x \in C_{\gamma, \epsilon}^{\alpha}[a, b]$ to problem (22) with the condition $x(a)=d_{0} \in \mathbb{R}$.

Proof Theorem 3.1 gives us the sufficiency to establish the existence of a unique solution $x \in C_{\gamma, \epsilon}^{\alpha, n-1}[a, b]$ to $(44)$.

First step: We show the existence of a unique solution $x \in C_{\gamma}^{n-1}[a, b]$.

(a) Choosing $t_{1} \in[a, b]$, we prove the existence of a unique solution $x \in C_{\gamma}^{n-1}\left[a, t_{1}\right]$ satisfying the conditions

$$
\sum_{k=0}^{n-1} \sum_{j=0}^{m} A_{j}\left(\frac{t_{1}^{\rho}-a^{\rho}}{\rho}\right)^{\operatorname{Re}\left(\alpha-\alpha_{j}\right)-k} \frac{\Gamma(1-\epsilon)}{\Gamma\left(1-\epsilon+\alpha-\alpha_{j}-k\right)}<1, \quad \epsilon \leq \alpha
$$

We then apply Theorem 2.6 to prove that there is a unique solution $x \in C_{\gamma}^{n-1}\left[a, t_{1}\right]$ to (44).

Equation (44) can be rewritten in the form $x(t)=(T x)(t)$, where

$$
(T x)(t)=\sum_{j=0}^{n-1} \frac{d_{j}}{j !}\left(\frac{t^{\rho}-a^{\rho}}{\rho}\right)^{j}+\frac{1}{\Gamma(\alpha)} \int_{a}^{t}\left(\frac{t^{\rho}-a^{\rho}}{\rho}\right)^{\alpha-1} h[\tau, \psi(\tau, x)] \frac{d \tau}{\tau^{1-\rho}}
$$

Denoting $x_{0}(t)=\sum_{j=0}^{n-1} \frac{d_{j}}{j !}\left(\frac{t^{\rho}-a^{\rho}}{\rho}\right)^{j}$, it follows that $x_{0}(t) \in C_{\gamma}^{n-1}\left[a, t_{1}\right]$ since $x_{0}(t)$ can be expressed as a finite sum of functions in $C_{\gamma}^{n-1}\left[a, t_{1}\right]$. Furthermore,

$$
h[\tau, \psi(\tau, x)] \in C_{\epsilon, \rho}[a, b] \quad \Longrightarrow \quad h[\tau, \psi(\tau, x)] \in C_{\epsilon, \rho}\left[a, t_{1}\right]
$$

and using equation (42), we obtain

$$
{ }_{a} I^{\alpha, \rho} h[\tau, \psi(\tau, x)](t) \in C\left[a, t_{1}\right] \quad \text { if } \epsilon \leq \alpha
$$

where $\alpha>0$ and $0 \leq \epsilon<1$.

Let $x \in C_{\gamma}^{n-1}\left[a, t_{1}\right]$, then using (43), it can be observed that the integral term on the righthand side of (44) belongs to $C_{\gamma}^{n-1}\left[a, t_{1}\right]$. This means ${ }_{a} I^{\alpha, \rho} h[\tau, \psi(\tau, x)](t) \in C_{\gamma}^{n-1}\left[a, t_{1}\right]$. Thus, $T x \in C_{\gamma}^{n-1}\left[a, t_{1}\right]$ and therefore we have proven that $T$ is continuous on $C_{\gamma}^{n-1}\left[a, t_{1}\right]$. Next we show that $T$ is a contraction by proving that given $x_{1}, x_{2} \in C_{\gamma}^{n-1}\left[a, t_{1}\right], \exists u>0$ such that

$$
\left\|T x_{1}-T x_{2}\right\|_{C_{\gamma}^{n-1}\left[a, t_{1}\right]} \leq u\left\|x_{1}-x_{2}\right\|_{C_{\gamma}^{n-1}\left[a, t_{1}\right]} .
$$


Using Lemma 2.1, Lemma 2.4, and (39), we obtain

$$
\begin{aligned}
&\left\|\left({ }_{a} I^{\alpha, \rho}\left(h\left[\tau, x_{1},{ }_{a}^{C} D^{\alpha_{1}, \rho} x_{1}, \ldots,{ }_{a}^{C} D^{\alpha_{m}, \rho} x_{1}\right]-h\left[\tau, x_{2},{ }_{a}^{C} D^{\alpha_{1}, \rho} x_{2}, \ldots,{ }_{a}^{C} D^{\alpha_{m}, \rho} x_{2}\right]\right)\right)\right\|_{C_{\gamma}^{n-1}\left[a, t_{1}\right]} \\
& \leq{ }_{a} I^{\alpha, \rho}\left(\| h\left[\tau, x_{1},{ }_{a}^{C} D^{\alpha_{1}, \rho} x_{1}, \ldots,{ }_{a}^{C} D^{\alpha_{m}, \rho} x_{1}\right]\right. \\
&\left.\quad-h\left[\tau, x_{2},{ }_{a}^{C} D^{\alpha_{1}, \rho} x_{2}, \ldots,{ }_{a}^{C} D^{\alpha_{m}, \rho} x_{2}\right] \|_{C_{\gamma}^{n-1}\left[a, t_{1}\right]}\right) \\
& \leq\left(\sum_{j=0}^{m} A_{j}\left\|\left(a_{a} I^{\alpha-\alpha_{j}, \rho}\right){ }_{a} I^{\alpha_{j}, \rho}\left({ }_{a}^{C} D^{\alpha_{j}, \rho}\right)\left(x_{1}-x_{2}\right)\right\|_{C_{\gamma}^{n-1}\left[a, t_{1}\right]}\right) \\
&=\left(\sum_{j=0}^{m} A_{j a} I^{\alpha-\alpha_{j}, \rho}\left\|_{a} I^{\alpha_{j}, \rho}\left({ }_{a}^{C} D^{\alpha_{j}, \rho}\right)\left(x_{1}-x_{2}\right)\right\|_{C_{\gamma}^{n-1}\left[a, t_{1}\right]}\right) \\
&= {\left[\left(\sum_{j=0}^{m} A_{j a} I^{\alpha-\alpha_{j}, \rho}\left\|x_{1}-x_{2}\right\|_{C_{\gamma}^{n-1}\left[a, t_{1}\right]}\right)(\tau)-\sum_{k_{j}=0}^{n_{j}-1} \frac{\gamma^{k_{j}}\left(x_{1}-x_{2}\right)(a)}{k_{j} !}\left(\frac{t^{\rho}-a^{\rho}}{\rho}\right)\right] . }
\end{aligned}
$$

Now, since $x_{1}, x_{2} \in C_{\gamma}^{n-1}\left[a, t_{1}\right]$, it follows that $\gamma^{k_{j}} x_{1}(a)=\gamma^{k_{j}} x_{2}(a)$, and therefore

$$
\begin{aligned}
& \left\|I_{a} I^{\alpha, \rho}\left(h\left[\tau, x_{1},{ }_{a}^{C} D^{\alpha_{1}, \rho} x_{1}, \ldots,{ }_{a}^{C} D^{\alpha_{m}, \rho} x_{1}\right]-h\left[\tau, x_{2},{ }_{a}^{C} D^{\alpha_{1}, \rho} x_{2}, \ldots,{ }_{a}^{C} D^{\alpha_{m}, \rho} x_{2}\right]\right)\right\|_{C_{\gamma}^{n-1}\left[a, t_{1}\right]} \\
& \quad \leq \sum_{j=0}^{m} A_{j}\left({ }_{a} I^{\alpha-\alpha_{j}, \rho}\left\|x_{1}-x_{2}\right\|\right)
\end{aligned}
$$

or

$$
\left\|a^{\alpha, \rho}\left(h\left[\tau, \psi\left(\tau, x_{1}\right)\right]-h\left[\tau, \psi\left(\tau, x_{2}\right)\right]\right)(t)\right\| \leq \sum_{j=0}^{m} A_{j}\left(a^{\alpha-\alpha_{j}, \rho}\left\|x_{1}-x_{2}\right\|\right)(t) .
$$

Then, according to the second part of Lemma 2.7 and equation (48), we have

$$
\begin{aligned}
& \left\|I^{\alpha, \rho}\left(h\left[\tau, \psi\left(\tau, x_{1}\right)\right]-h\left[\tau, \psi\left(\tau, x_{2}\right)\right]\right)(t)\right\|_{C_{\gamma}^{n-1}\left[a, t_{1}\right]} \\
& \leq\left\|\sum_{k=0}^{n-1} I^{\alpha-k, \rho}\left(h\left[\tau, \psi\left(\tau, x_{1}\right)\right]-h\left[\tau, \psi\left(\tau, x_{2}\right)\right]\right)(t)\right\|_{C_{\gamma}\left[a, t_{1}\right]} \\
& \quad \leq \sum_{k=0}^{n-1} \sum_{j=0}^{m} A_{j}\left(\frac{t_{1}^{\rho}-a^{\rho}}{\rho}\right)^{\operatorname{Re}\left(\alpha-\alpha_{j}\right)-k} \frac{\Gamma(1-\epsilon)}{\Gamma\left(1-\epsilon+\alpha-\alpha_{j}-k\right)}\left\|x_{1}-x_{2}\right\|_{C_{\gamma}^{n-1}\left[a, t_{1}\right]} .
\end{aligned}
$$

Hence

$$
\left\|T x_{1}-T x_{2}\right\|_{C_{\gamma}^{n-1}\left[a, t_{1}\right]} \leq u\left\|x_{1}-x_{2}\right\|_{C_{\gamma}^{n-1}\left[a, t_{1}\right]} \quad \forall x_{1}, x_{2} \in C_{\gamma}^{n-1}\left[a, t_{1}\right]
$$

This tells us that there is a fixed point $x_{f}^{*} \in_{C_{\gamma}^{n-1}\left[a, t_{1}\right]}$ which is defined explicitly as a limit of iterations of the mapping $T$. That is,

$$
\lim _{p \rightarrow \infty}\left\|x_{p}(t)-x^{*}(t)\right\|_{C_{\gamma}^{n-1}[a, b]}=0
$$


where

$$
x_{p}(t)=T^{p} x_{f_{i}}^{*}, \quad x_{f_{i}}^{*}(t)=x_{f}(t), \quad x^{*}(t)=x_{i}^{*}(t), \quad i=0,1, \ldots, M,
$$

and

$$
x_{i}^{*}\left(t_{i+1}\right)=x_{i+1}^{*}\left(t_{i+1}\right), \quad[a, b]=\bigcup\left[t_{i}, t_{i+1}\right], \quad a=t_{0}<\cdots<t_{m}=m .
$$

\section{Second step:}

It should be noted that proving the unique solution $x^{*}(t)$ belonging to $C_{\gamma, \epsilon}^{\alpha, n-1}[a, b]$ completes the proof. Then it suffices to show that $\left({ }_{a}^{C} D^{\alpha, \rho} x\right)(t) \in C_{\epsilon, \rho}[a, b]$.

From (48),

$$
\begin{aligned}
& \left\|\left({ }_{a}^{C} D^{\alpha, \rho} x_{p}\right)(t)-\left({ }_{a}^{C} D^{\alpha, \rho} x^{*}\right)(t)\right\|_{C_{\epsilon, \rho}[a, b]} \\
& =\left\|h\left[t, \psi\left(t, x_{p}\right)\right]-h\left[t, \psi\left(t, x^{*}\right)\right]\right\|_{C_{\epsilon, \rho}[a, b]} \\
& \leq \sum_{j=0}^{m} A_{j}\left\|_{a}^{C} D^{\alpha, \rho}\left(x_{p}(t)-x^{*}(t)\right)\right\|_{C_{\epsilon, \rho}[a, b]} \\
& \leq \sum_{j=0}^{m} A_{j}\left\|_{a} I^{n-1-\alpha_{j}, \rho} \gamma^{n-1}\left(x_{p}(t)-x^{*}(t)\right)\right\|_{C_{\epsilon, \rho}[a, b]} \\
& \leq \sum_{j=0}^{m} A_{j}\left(\frac{b^{\rho}-a^{\rho}}{\rho}\right)\left\|_{a}^{\epsilon} I^{n-1-\alpha_{j}, \rho} \gamma^{n-1}\left(x_{p}(t)-x^{*}(t)\right)\right\|_{C[a, b]} \\
& \leq \sum_{j=0}^{m} A_{j} \frac{\left(\frac{b^{\rho}-a^{\rho}}{\rho}\right)^{\epsilon}}{\Gamma\left(n-\alpha_{j}\right)}\left\|\gamma^{n-1}\left(x_{p}(t)-x^{*}(t)\right)\right\|_{C[a, b]} \\
& \leq \sum_{j=0}^{m} A_{j} \frac{\left(\frac{b^{\rho}-a^{\rho}}{\rho}\right)^{\epsilon}}{\Gamma\left(n-\alpha_{j}\right)}\left\|\left(x_{p}(t)-x^{*}(t)\right)\right\|_{C^{n-1}[a, b]} .
\end{aligned}
$$

Taking limit as $p \rightarrow \infty$ makes the right-hand side of the above inequality approach 0 independently. This implies

$$
\lim _{p \rightarrow \infty}\left\|\left({ }_{a}^{C} D^{\alpha, \rho} x_{p}\right)(t)-\left({ }_{a}^{C} D^{\alpha, \rho} x^{*}\right)(t)\right\|_{C_{\epsilon, \rho}[a, b]}=0 .
$$

Hence, there exists a unique solution $x^{*} \in C_{\gamma, \epsilon}^{\alpha, n-1}[a, b]$ to equation (44).

(b) In the same way, the second part of the theorem can be proven.

Corollary 3.3 When $\epsilon=0$ and with the assumptions of Theorem 3.2, a unique solution $x^{*}(t) \in C_{\gamma}^{n-1}[a, b]$ to problem (1)-(2) exists.

Proof The proof is analogous to that of Theorem 3.2 where

$$
\begin{aligned}
& \left\|I^{\alpha, \rho}\left(h\left[\tau, \psi\left(\tau, x_{1}\right)\right]-h\left[\tau, \psi\left(\tau, x_{2}\right)\right]\right)(t)\right\|_{C\left[t_{i}, t_{i+1}\right]} \\
& \quad \leq \sum_{k=0}^{n-1} \sum_{j=0}^{m} A_{j} \frac{\left(\frac{t_{i+1} \rho-t_{i} \rho}{\rho}\right)^{\left(\alpha-\alpha_{j}\right)-k}}{\Gamma\left(\alpha-\alpha_{j}-k+1\right)} \times\left\|\left(x_{1}(t)-x_{2}(t)\right)\right\|_{C\left[t_{i}, t_{i+1}\right]},
\end{aligned}
$$




$$
\begin{aligned}
& i=0,1, \ldots, M \text { with } t_{0}=a, t_{M}=b \text { and } \\
& \qquad\left\|\left({ }_{a}^{C} D^{\alpha, \rho} x_{p}\right)(t)-\left({ }_{a}^{C} D^{\alpha, \rho} x^{*}\right)(t)\right\|_{C_{\epsilon, \rho}[a, b]} \\
& \quad \leq \sum_{j=0}^{m} A_{j} \frac{\left(\frac{b^{\rho}-a^{\rho}}{\rho}\right)^{\epsilon}}{\Gamma\left(n-\alpha_{j}\right)} \times\left\|\left(x_{p}(t)-x^{*}(t)\right)\right\|_{C^{n-1}[a, b]} .
\end{aligned}
$$

Corollary 3.4 Let $\alpha>0$ with $n=[\alpha]+1$ and $0 \leq \epsilon<1$ such that $\epsilon \leq \alpha$. For positivenon-zero integer $m$, if $f(t) \in C_{\epsilon, \rho}[a, b], d_{j}(t) \in C[a, b]$, and $\alpha_{j}>0(j=1, \ldots, m)$ satisfying (45), then there exists a unique solution $x(t) \in C_{\gamma}^{\alpha, n-1}[a, b]$ to the Cauchy problem for the following linear fractional differential equation of order $\alpha$ :

$$
\left({ }_{a}^{C} D^{\alpha, \rho} x\right)(t)+\sum_{j=1}^{m} d_{j}(t)\left({ }_{a}^{C} D^{\alpha_{j}, \rho} x\right)(t)+d_{0}(t) x(t)=f(t), \quad t>a
$$

\section{having initial conditions (2).}

Proof The proof follows immediately from Theorem 3.2.

\section{Acknowledgements}

The fourth author would like to thank Prince Sultan University for funding this work through research group Nonlinear Analysis Methods in Applied Mathematics (NAMAM) group number RG-DES-2017-01-17.

\section{Competing interests}

The authors declare they have no competing interests.

\section{Authors' contributions}

The main idea of this paper was proposed by TA and FJ. YYG and RA prepared the manuscript and performed all the steps of the proofs equally in this research. All authors read and approved the final manuscript.

\section{Author details}

${ }^{1}$ Department of Mathematics, Faculty of Sciences, Northwest University Kano, Kano, Nigeria. ${ }^{2}$ Department of Mathematics, Selçuk University, Konya, Turkey. ${ }^{3}$ Department of Mathematics, Faculty of Arts and Sciences, Cankaya University, Ankara, Turkey. ${ }^{4}$ Department of Mathematics and General Sciences, Prince Sultan University, Riyadh, Saudi Arabia.

\section{Publisher's Note}

Springer Nature remains neutral with regard to jurisdictional claims in published maps and institutional affiliations.

Received: 5 January 2018 Accepted: 8 April 2018 Published online: 13 April 2018

\section{References}

1. Jarad, F., Abdeljawad, T., Baleanu, D.: On the generalized fractional derivatives and their Caputo modification. J. Nonlinear Sci. Appl. 10, 2607-2619 (2017)

2. Podlubny, I.: Fractional Differential Equations. Academic Press, San Diego (1999)

3. Samko, S.G., Kilbas, A.A., Marichev, O.I.: Fractional Integrals and Derivatives: Theory and Applications. Gordon and Breach, Yverdon (1993)

4. Kilbas, A., Srivastava, H.M., Trujillo, J.J.: Theory and Application of Fractional Differential Equations. North Holland Mathematics Studies, vol. 204. Elsevier, Amsterdam (2006)

5. Magin, R.L.: Fractional Calculus in Bioengineering. Begell House Publishers, Redding (2006)

6. Machado, J.A.T., Kiryakova, V., Mainardi, F.: Recent history of fractional calculus. Commun. Nonlinear Sci. Numer. Simul. 16(3), 1140-1153 (2011)

7. Hilfer, R.: Applications of Fractional Calculus in Physics. Word Scientific, Singapore (2000)

8. Lorenzo, C.F., Hartley, T.T.: Variable order and distributed order fractional operators. Nonlinear Dyn. 29, 57-98 (2002)

9. Kilbas, A.A.: Hadamard type fractional calculus. J. Korean Math. Soc. 38, 1191-1204 (2001)

10. Katugampola, U.N.: New approach to generalized fractional integral. Appl. Math. Comput. 218, 860-865 (2011)

11. Katugampola, U.N.: A new approach to generalized fractional derivatives. Bull. Math. Anal. Appl. 6(4), 1-15 (2014)

12. Jarad, F., Abdeljawad, T., Baleanu, D.: Caputo-type modification of the Hadamard fractional derivative. Adv. Differ. Equ. 2012, 142 (2012). https://doi.org/10.1186/1687-1847-2014-10

13. Jarad, Y.Y.G.F., Abdeljawad, T., Baleanu, D.: On Caputo modification of the Hadamard fractional derivative. Adv. Differ. Equ. 2014, 10 (2014). https://doi.org/10.1186/1687-1847-2012-142 
14. Jarad, F., Uğurlu, E., Abdeljawad, T., Baleanu, D.: On a new class of fractional operators. Adv. Differ. Equ. 2017, 247 (2017)

15. Caputo, M., Fabrizio, M.: A new definition of fractional derivative without singular kernel. Prog. Fract. Differ. Appl. 1(2), 73-85 (2015)

16. Atangana, A., Baleanu, D.: New fractional derivative with non-local and non-singular kernel. Therm. Sci. 20(2), 757-763 (2016)

17. Yang, X.J., Srivastava, H.M., Machado, J.A.T.: A new fractional derivatives without singular kernel: application to the modelling of the steady heat flow. Therm. Sci. 20, 753-756 (2016)

18. da Vanterler, J., Sousa, C., Capelas de Oliveira, E.: On the $\psi$-Hilfer fractional derivative. Commun. Nonlinear Sci. Numer. Simul. 60, 72-91 (2018)

19. da Vanterler, J., Sousa, C., Capelas de Oliveira, E.: A Gronwall inequality and the Cauchy-type problem by means of $\psi$-Hilfer operator (2017) arXiv:1709.03634v1

20. da Vanterler, J., Sousa, C., Capelas de Oliveira, E.: A new fractional derivative of variable order with non-singular order and fractional differential equations (2017) arXiv:1712.06506v1

21. Oliveira, D.S., Capelas de Oliveira, E.: On A Caputo-type fractional derivatives. Advances in Pure and Applied Mathematics. Accepted for publication

22. Daftardar-Gejji, V., Jaffari, H.: Analysis of a system of nonautonomous fractional differential equations involving Caputo derivatives. J. Math. Anal. Appl. 328, 1026-1033 (2007)

23. Delbosco, D., Rodino, L.: Existence and uniqueness for a nonlinear fractional differential equation. J. Math. Anal. Appl. 204, 609-625 (1996)

24. Abdeljawad, T., Baleanu, D., Jarad, F.: Existence and uniqueness theorem for a class of delay differential equations with left and right Caputo fractional derivatives. J. Math. Phys. 49(8), 083507 (2018)

25. Abdeljawad, T., Jarad, F., Baleanu, D.: On the existence and the uniqueness theorem for fractional differential equations with bounded delay within Caputo derivatives. Sci. China Ser. A 51(10), 1775-1786 (2008)

26. Adjabi, Y., Jarad, F., Baleanu, D., Abdeljawad, T.: On Cauchy problems with Caputo-Hadamard fractional derivatives. J. Comput. Anal. Appl. 21(4), 661-681 (2016)

27. Adjabi, Y., Jarad, F., Abdeljawad, T.: On generalized fractional operators and a Gronwall type inequality with applications. Filomat 31(17), 5457-5473 (2017)

\section{Submit your manuscript to a SpringerOpen ${ }^{\circ}$ journal and benefit from:}

- Convenient online submission

- Rigorous peer review

- Open access: articles freely available online

- High visibility within the field

- Retaining the copyright to your article

Submit your next manuscript at $\gg$ springeropen.com 